\title{
GHGT-9
}

\section{Measurements of Non-Wetting Phase Trapping Applied to Carbon Dioxide Storage}

\author{
Saleh Al Mansoori ${ }^{\mathrm{a}, *}$, Stefan Iglauer ${ }^{\mathrm{a}}$, Christopher H Pentland $^{\mathrm{a}}$, \\ Branko Bijeljic $^{\mathrm{a}}$, Martin J Blunt ${ }^{\mathrm{a}}$ \\ ${ }^{a}$ Department of Earth Science and Engineering, Imperial College, London SW7 2AZ UK
}

\begin{abstract}
We measure the trapped non-wetting phase saturation as a function of the initial saturation in sand packs. The application of the work is for carbon dioxide $\left(\mathrm{CO}_{2}\right)$ storage in aquifers where capillary trapping is a rapid and effective mechanism to render injected $\mathrm{CO}_{2}$ immobile. We used analogue fluids at ambient conditions. The trapped saturation initially rises linearly with initial saturation to a value of 0.11 for oil/water systems and 0.14 for gas/water systems. There then follows a region where the residual saturation is constant with further increases in initial saturation.
\end{abstract}

(c) 2009 Elsevier Ltd. All rights reserved.

Non-wetting phase, carbon dioxide, saturation, super-critical, climate change.

\section{Introduction}

If we are to avoid potentially dangerous climate change, we need to capture and store $\mathrm{CO}_{2}$ emitted by fossil-fuel burning power stations and other industrial plants [1]. Saline aquifers provide the largest potential for storage and the widest geographical spread [2]. Subsequent leakage of $\mathrm{CO}_{2}$ into the atmosphere, even over hundreds of years, would render any sequestration scheme inefficient. However, based on the experience of the oil and gas industry, there is a good understanding of trapping mechanisms that take place in geological formations.

Hydrodynamic trapping is the primary mechanism by which hydrocarbons accumulate in the subsurface. The same mechanism would take place during carbon sequestration, with the less dense $\mathrm{CO}_{2}$ rising due to buoyancy forces until it is trapped under impermeable cap-rock [3]. However, this process relies on there being an intact barrier to upwards flow. Solution trapping occurs because of dissolution of $\mathrm{CO}_{2}$ in the aquifer brine. The $\mathrm{CO}_{2}$ saturated brine is denser than the surrounding brine leading to convective mixing where the denser brine migrates

* Corresponding author. Tel.: +44 (0) 20759 47137; fax: +44 (0)20 75947403.

E-mail address: s.al-mansoori@ ic.ac.uk 
deeper into the formation [4-6]. Mineral trapping occurs when $\mathrm{CO}_{2}$ dissolves in formation brine which subsequently reacts with the host rock to generate solid minerals over periods of thousands to billions of years [7-10].

The final trapping mechanism involves $\mathrm{CO}_{2}$ becoming immobile at the pore scale by capillary forces. This process occurs as the $\mathrm{CO}_{2}$ migrates upwards, when it is displaced by natural groundwater flow or by the injection of chase brine. It is a rapid and effective trapping mechanism that reduces the need to ensure cap-rock integrity [11$16]$.

\section{Capillary Trapping}

Capillary trapping has been measured in oil-water, gas-water and three-phase gas-water-oil systems; Figure 1 shows a compilation of two-phase data in the literature [17-31].

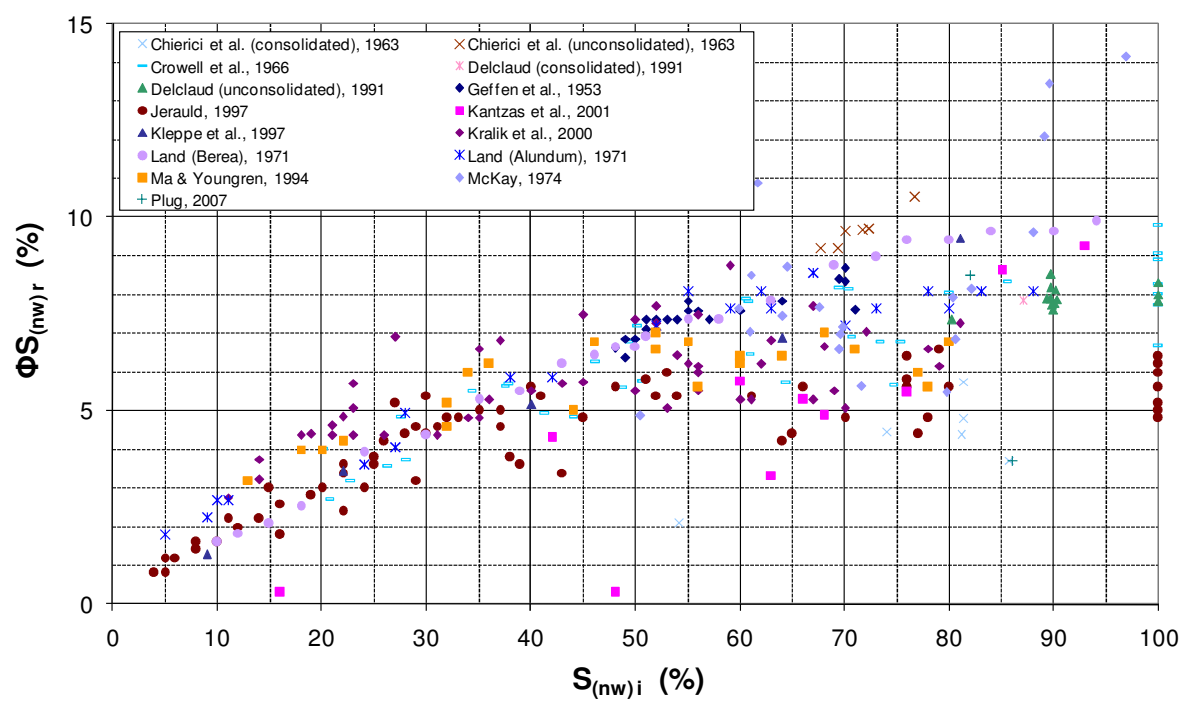

Figure 1. Database of trapping capacity $\phi S_{(n w) r}$ versus initial non-wetting phase saturation $S_{(n w) i}$ in the literature.

We define the trapping capacity as the fraction of the total volume of the porous medium that can be occupied by the trapped residual non-wetting phase saturation: $\phi S_{(n w) r}$, where $\phi$ is the porosity and $S_{(n w) r}$ is the residual saturation. The trapping capacity increases approximately linearly with initial saturation until an initial saturation of around $50 \%$. Beyond this there is considerable scatter, with most of the data indicating a maximum trapping capacity of between 4 and $10 \%$.

\section{Experimental Methodology and Equipment}

Our experiments used unconsolidated sand packs and analogue fluids at ambient conditions. The fluid systems investigated were oil/water and gas/water. Above its critical point - critical temperature $=304.45 \mathrm{~K}$; critical pressure $=7.39 \mathrm{MPa}\left(\right.$ IPCC, 2005) $-\mathrm{CO}_{2}$ has unique properties with a density similar to a liquid and a viscosity more akin to a gas. For the oil/water systems, the non-wetting phase was chosen to be n-octane since it has a density similar to $\mathrm{CO}_{2}$ at reservoir conditions. Air was used as the non-wetting phase for the gas/water systems which has a viscosity similar to the viscosity of $\mathrm{CO}_{2}$ at reservoir conditions. The wetting phase in both sets of experiments was brine (de-ionized water with 1 weight \% Potassium Chloride, and 5 weight \% Sodium Chloride). The ambient temperature in the lab was recorded as being consistently $293.15 \mathrm{~K}( \pm 1 \mathrm{~K})$. Ambient pressure was measured to be 
$0.101 \mathrm{MPa}( \pm 0.003 \mathrm{MPa})$. The sand used in the experiments was unconsolidated Levenseat 60 (LV60) sand (WBB Minerals, UK).

The sand pack was contained in a custom machined Polymethylmethacrylate column $1 \mathrm{~m}$ long. The design of the column features pre-machined grooves around the circumference every $5 \mathrm{~cm}$ along the column length. These grooves allowed the column to be sliced upon completion of the flow experiment such that direct samples could be taken from the sand pack for analysis using gas chromatography (GC) (oil/water systems) or mass balance (air/water systems). When packing the column with sand, a circular piece of wire mesh (brass) and two circular pieces of filter paper (VWR Int. Filter Papers 415) were placed between the sand face and the end cap to prevent sand production. A balance Model S-6002 (Denver Instruments) was used to measure the mass of the sand-packed column in different conditions, dry, saturated and sliced (accuracy of $\pm 1.0 \times 10^{-5} \mathrm{~kg}$ ).

The use of GC for direct sample measurement [32] is a precise approach that is highly accurate. A PerkinElmer Autosystem XL GC fitted with a SGE forte capillary column $(30 \mathrm{~m} \times 0.25 \mathrm{~mm}$ ID, BP20 $0.5 \mu \mathrm{m}$, polyethylene glycol) and a thermal conductivity detector (TCD) was used to detect fluid concentrations. The GC set-up allowed detection of changes of the magnitude of $1 \mathrm{ppm}$ within each GC sample vial. A high precision syringe pump (Teledyne Pump 1000D) was used in all flooding experiments. Flow accuracy according to technical specifications is $\pm 0.5 \%$.

In total four experiments were performed for the oil/water system. Each experiment consisted of one set of $S_{o i}$ measurements and one set of $S_{o r}$ measurements. In three experiments octane was injected into a vertical column, while the fourth used horizontal injection. In each experiment the volume of octane injected varied to investigate the full range of initial oil saturations. In two experiments three replicates were performed to find both $S_{o i}$ and $S_{o r}$. For the gas/water system, three $S_{g i}$ and two $S_{g r}$ experiments were performed. In all experiments the air was allowed to enter the top of the column in a gravity drainage process.

\section{Experimental Procedure}

The experimental procedures were as follows:

\subsection{Oil/Water System:}

1. Column dry packed with LV60 sand.

2. Column fully saturated with brine.

3. Octane injection (primary drainage) - performed to reach $S_{o i}$.

4. Brine injection (secondary imbibition) - performed to reach $S_{o r}$.

5. Column sliced into eighteen sections for sampling.

6. Addition of solvent to each sample of sand + n-octane + brine.

7. Filtering of each sample removes sand leaving a single homogeneous liquid phase.

8. Injection of homogeneous liquid samples into GC for compositional analysis.

\subsection{Gas/Water System:}

1. Repeat steps 1 and 2 in section 4.1.

2. Air injection (primary drainage) - performed to reach $S_{g i}$.

3. Brine injection (secondary imbibition) - performed to reach $S_{g r}$.

4. Column sliced into eighteen sections.

5. Weigh each column section filled with sand, brine and air.

6. Recover sand and wash with de-ionized water and measure sand mass.

7. Weigh each empty clean column section.

8. Measure bulk volume of each column section. 
Table 1. Average properties of an LV60 sand pack.

\begin{tabular}{|l|l|}
\hline Porosity & $37 \% \pm 0.2 \%$ \\
\hline Permeability & $32 \mathrm{D} \pm 0.3 \mathrm{D}$ \\
\hline Pore Volume & $111.2 \mathrm{ml} \pm 2.7 \mathrm{ml}$ \\
\hline Formation Factor & 4.8 \\
\hline
\end{tabular}

Regardless of the fluid system the procedure began with the packing of a column with LV60 sand. Each individual sand-packed column would yield either an $S_{o i} / S_{o r}$ or an $S_{g i} / S_{g r}$ set of data. Two columns - as a minimum were required to produce a single set of $S_{o i}$ versus $S_{o r}$ (or $S_{g i}$ versus $S_{g r}$ ) values; we added additional replicates to assess the reproducibility of the results.

A known mass of sand was added to a column with a known bulk volume. The subsequent porosity of the sand pack was determined by mass balance. A packing density of $1654 \mathrm{~kg} / \mathrm{m}^{3}$ was used throughout. The bulk volume of the columns was found by filling them with water of a known density and measuring the mass and comparing with the mass of the dry column. The packing process was performed with dry sand poured into the column in one continuous motion under vibration. Table 1 displays the average properties of the packs.

Establishing an initial brine saturation of $100 \%$ was accomplished in three steps. The brine was completely deaired by bubbling oxygen-free nitrogen gas through the brine for a minimum of 15 minutes. In addition, and prior to brine injection, the column was completely saturated with gaseous $\mathrm{CO}_{2} .500 \mathrm{ml}$ of brine was injected under a 0.138 MPa back-pressure to displace the $\mathrm{CO}_{2}$. The back-pressure was applied with a needle valve, forcing the brine into the smaller pores. Even so any remaining trapped $\mathrm{CO}_{2}$ gas dissolved in the brine, resulting in a $100 \%$ saturated sand pack.

For the oil/water system three vertical experiments were performed, with different volumes of octane injected in each case. The octane was injected into the top of a column which was positioned vertically. Once the desired volume of octane had been injected, the column was inverted such that the high oil saturation interval was at the bottom. The octane subsequently migrated upwards due to buoyancy forces. Oil migration was stopped by placing the column horizontally once the first drop of octane reached the filter paper at the top end of the column. In the air/brine experiments, the inlet and outlet at the top and bottom of the column were opened so that air migrated into the sandpack by gravity forces for 3.5 hours.

The column would either be sliced to find $S_{o i} / S_{g i}$ or brine injection would start. To reach residual conditions 450 $\mathrm{ml}$ of brine, four pore volumes, were injected. In the case of the oil/water system oil production always ceased soon after brine breakthrough and long before the end of the experiment.

In the oil/water system the experimental procedure replicates a situation where injected $\mathrm{CO}_{2}$ migrates upwards due to buoyancy forces before being trapped as a residual phase by chase brine. This is true for the portion of the column uninvaded by oil at the point of flipping when buoyancy driven migration begins. The results from this portion of the column were of primary interest; however, as will be seen, the results from the entire length of the column describe a consistent trend and as such are included here.

For the oil/water experiments, in addition to the vertical experiments described above, an additional horizontal experiment was performed to investigate the effect of gravity. $500 \mathrm{ml}$ of octane was injected into a fully brinesaturated column positioned horizontally. The column was then sliced to find $S_{o i}$ or brine injection was started, following the procedure described above for the vertical experiment.

At the end of injection the columns were sliced into eighteen sample sections. For the oil/water system each section was mixed with $10 \mathrm{~g}$ of 2-propanol solvent. This amount of solvent had been determined to be sufficient to dissolve both the n-octane and brine giving a single homogeneous liquid phase. The mixture of sand and liquid was 
then filtered to remove the sand. The liquid was sampled into vials ready for GC analysis. This process was repeated for each of the eighteen samples from each column.

For the air/brine experiments, each sliced section was weighed full and empty. Wet sand contained in each section was washed three times with de-ionized water to remove dissolved salt. The sand was then dried and weighed. The bulk volume of each column section was measured. This step improved the accuracy of the results significantly as the pore volume for each $5 \mathrm{~cm}$ section was determined.

\section{Results}

Figure 2 shows the measured oil saturation profiles. The experiments cover different ranges of $S_{o i}$ : in experiment 1 it ranges from $1.6 \%$ to $49.9 \% ; 1.1 \%$ to $73.9 \%$ in experiment $2 ; 50.1 \%$ to $78.9 \%$ in experiment 3 ; and $70.0 \%$ to $80.4 \%$ for experiment 4 .

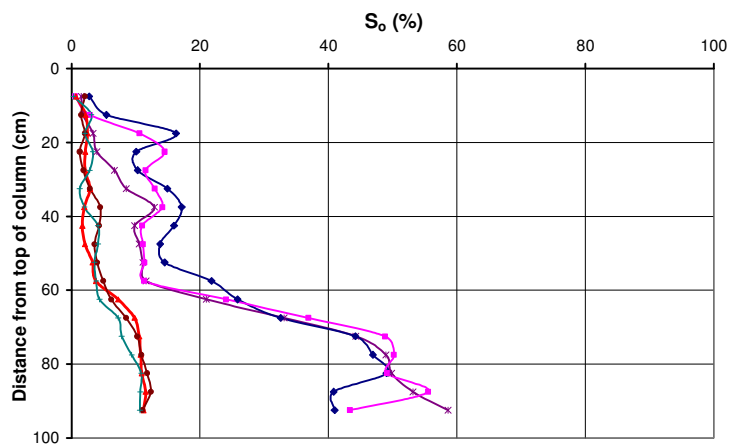

(a)

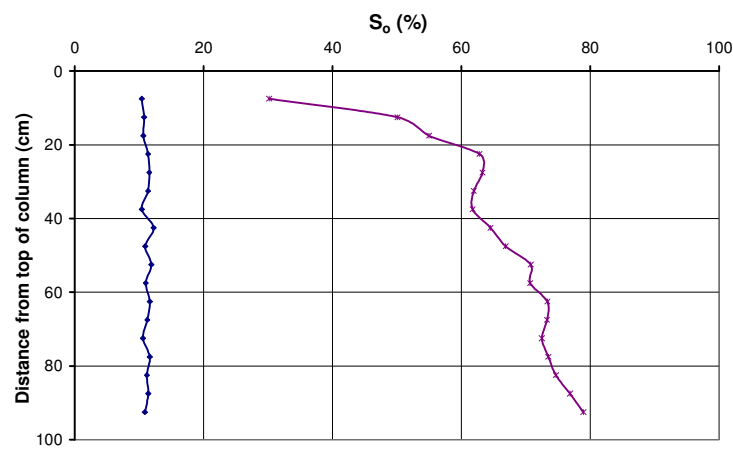

(c)

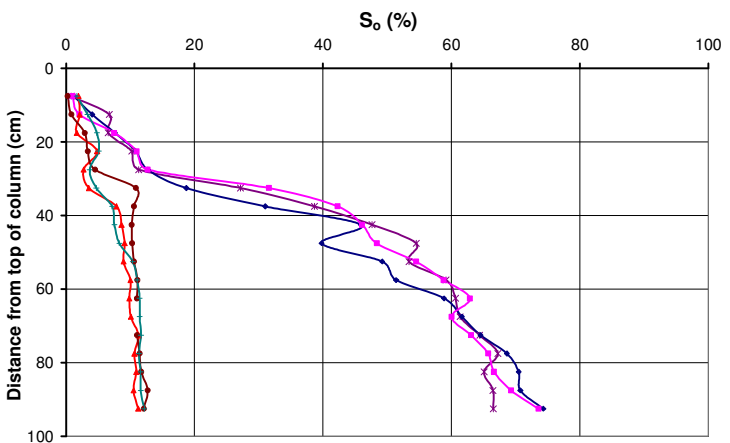

(b)

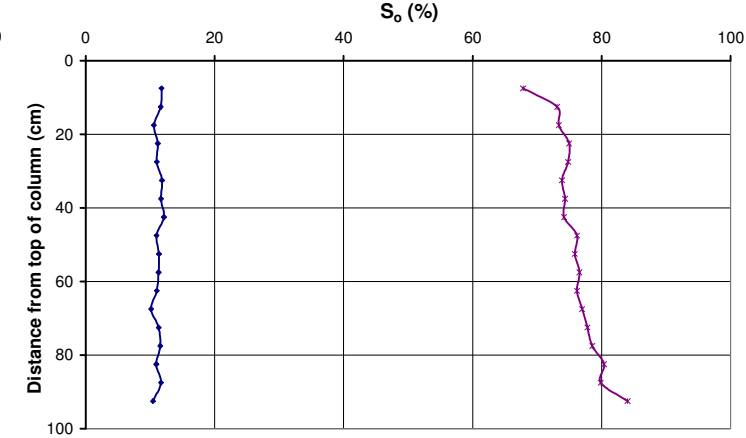

(d)

Figure 2. Oil Saturation profiles. The lines to the right show the initial conditions, while the lines to the left show the trapped oil saturation.

(a) Experiment 1: vertical $30 \mathrm{ml}$ oil injection. Three Soi replicates and three Sor replicates are shown.

(b) Experiment 2: vertical $50 \mathrm{ml}$ oil Injection. Three Soi replicates and three Sor replicates are shown.

(c) Experiment 3: vertical $80 \mathrm{ml}$ oil injection.

(d) Experiment 4: horizontal $500 \mathrm{ml}$ oil injection. 
Figure 3 shows the measured gas saturation profiles where $S_{g i}$ ranges from $9.3 \%$ to $74.4 \%$.

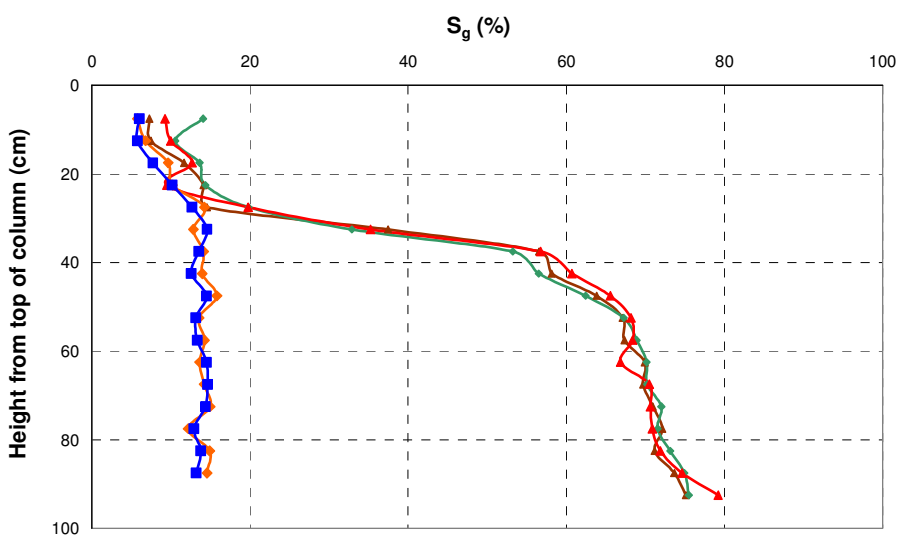

Figure 3. Gas Saturation profiles. The lines to the right show the initial conditions, while the lines to the left show the trapped gas saturation.

Figure 4 shows the measured trapping curves. The error bars in Figure 2 are based on the standard deviation from experiments where replicates were performed for each of the data sets. A good fit to the data is given by Aissaoui's correlation [33], with an initial linear increase in $S_{o r}$ to $S_{o r}^{\max }=11.3 \%$ at $S_{o c}=50 \%$ by constant $S_{o r}$ for an oil/water system; and a linear increase in $S_{g r}$ to $S_{g r}^{\max }=14 \%$ at $\mathrm{S}_{\mathrm{cg}}=20 \%$ followed by a constant $S_{g r}$ for a gas/water system.

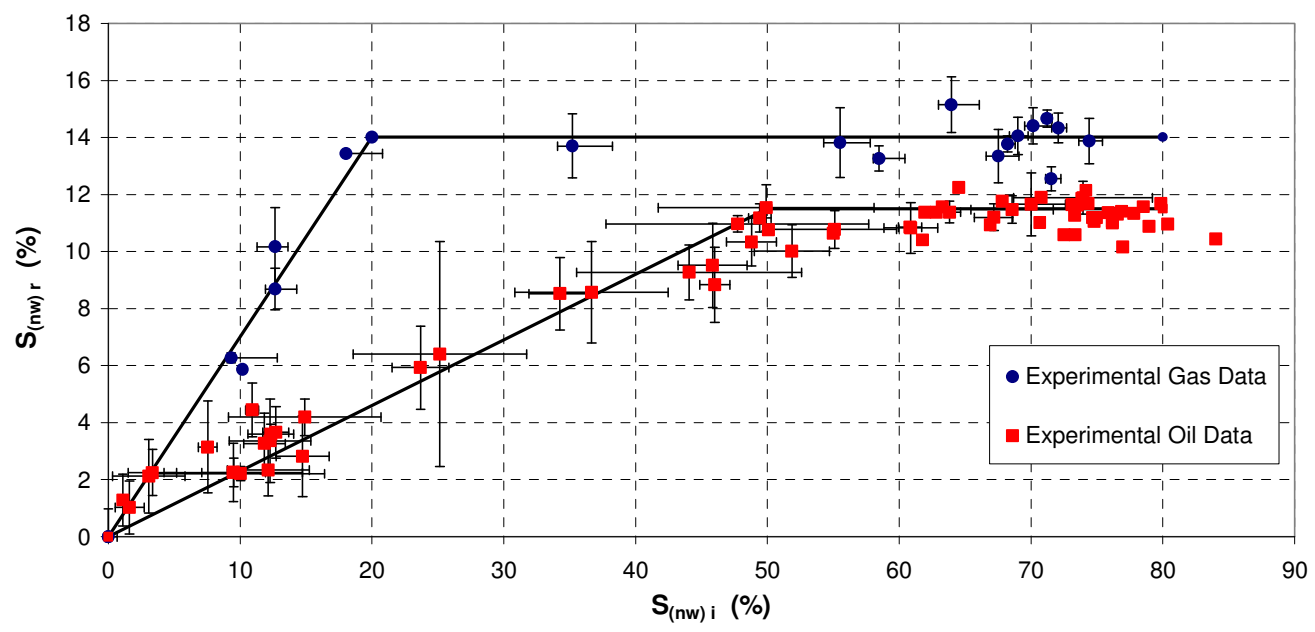

Figure 4. Comparison between the experimental gas and oil trapping data.

\section{Discussion and Conclusions}

We have measured the trend of both residual oil and residual gas saturations against initial saturations in unconsolidated sand packs. We found relatively low residual saturations; in the high-porosity homogeneous pore space, waterflooding at the pore scale proceeds as a uniform advance controlled by cooperative pore filling with 
little snap-off and trapping [34]. The residual saturations increased linearly with initial saturation until a maximum of $11.3 \%$ for oil and $14 \%$ for gas was reached for initial saturation of approximately $50 \%$ and $20 \%$ respectively. The observed trapping capacities were $\phi S_{o r}=4.1 \%$ and $\phi S_{g r}=5.2 \%$. The residual saturation does not increase as the initial saturation is increased further. At $50 \%$ oil (20\% gas) saturation, the non-wetting phase is able to occupy all the larger pore spaces in which it will be trapped during waterflooding - invasion of smaller pores during primary drainage does not increase the residual saturation since the non-wetting phase will be displaced from these regions. This is in contrast to highly consolidated media with a wide pore size distribution, where the non-wetting phase can be trapped in small pores that are only filled at high initial saturations. The Land trapping model [25] does not give good predictions of the trapped saturation for unconsolidated media; instead the linear Aissaoui's correlation [33] is a good match to the data.

This work implies that $\mathrm{CO}_{2}$ injection in poorly consolidated media would lead to rather poor storage efficiencies, with at most $4-6 \%$ of the rock volume occupied by trapped $\mathrm{CO}_{2}$; this is at the lower end of the compilation of literature results shown in Figure 1. Using the Land correlation to predict the behavior would tend to over-estimate the degree of trapping except for high initial saturations.

\section{Acknowledgements}

We would like to acknowledge funding from the following agencies: Shell under the Grand Challenge on Clean Fossil Fuels; Qatar Petroleum, Shell and the Qatar Science and Technology Park under the Qatar Carbonates and Carbon Storage Research Centre; the EPSRC (grant number EP/C536754/1); NERC (grant number NE/C516401/1); and ADNOC (Abu Dhabi).

\section{References}

1. Orr, J.F.M., Storage of Carbon Dioxide in Geologic Formations. Journal of Petroleum Technology, 2004. 56(9).

2. Hawkes, C.D., P.J. Mclellan, and S. Bachu, Geomechanical Factors Affecting Geological Storage of CO in Depleted Oil and Gas Reservoirs. Journal of Canadian Petroleum Technology, 2005. 44(10): p. 52-61.

3. Bachu, S., W.D. Gunter, and E.H. Perkins, Aquifer disposal of CO2 - hydrodynamic and mineral trapping. Energy Conversion \& Management, 1994. 35(4): p. 269-279.

4. Ennis-King, J. and L. Paterson, Role of convective mixing in the long-term storage of carbon dioxide in deep saline formations. SPE Journal, 2005. 10(3): p. 349-356.

5. Lindeberg, E. and D. Wessel-Berg, Vertical convection in an aquifer column under a gas cap of CO2. Energy Conversion Management,, 1997. 38(Supplement 1): p. S229-S234.

6. Riaz, A., et al., Onset of convection in a gravitationally unstable diffusive boundary layer in porous media. J. Fluid Mech, 2006. 548: p. 87-111.

7. Egermann, P., B. Bazin, and O. Vizika, An Experimental Investigation of Reaction-Transport Phenomena During CO Injection SPE 93674 presented at the 14th SPE Middle East Oil \& Gas Show and Conference held in Bahrain International Exhibition Center, Bahrain, 12-15 March 2005., 2005.

8. Gunter, W.D. and E.H. Perkins, Aquifer disposal of CO2 rich gases: reaction design for added capacity. Energy Conversion and Management, 1993. 34(9-11): p. 941-948.

9. Gunter, W.D., B. Wiwchar, and E.H. Perkins, Aquifer disposal of CO2-rich greenhouse gases: Extension of the time scale of experiment for CO2-sequestering reactions by geochemical modelling. Mineralogy and Petrology, 1997. 59(12): p. 121-140.

10. Lin, H., et al., Experimental evaluation of interactions in supercritical CO2/water/rock minerals system under geologic CO2 sequestration conditions. Journal of Material Science, 2008. 43(7): p. 2307-2315.

11. Hesse, M.A., H.A. Tchelepi, and F.M. Orr Jr. Scaling Analysis of the Migration of $\mathrm{CO}_{2}$ in Saline Aquifers. in Annual Technical Conference and Exhibition. 2006. San Antonio, Texas, USA., 24-27 SPE 102796, proceedings of the SPE.

12. Juanes, R., et al., Impact of relative permeability hysteresis on geological CO2 storage. Water Resources Research, 2006. 42(W12418, doi:10.1029/2005WR004806). 
13. Kumar, A., et al., Reservoir Simulation of $\mathrm{CO}_{2}$ Storage in Deep Saline Aquifers. SPE Journal, 2005. 10(3): p. $336-348$.

14. Obi, E.-O.I. and M.J. Blunt, Streamline-based simulation of carbon dioxide storage in a North Sea aquifer. Water Resources Research, 2006. 42(W03414 doi: 10.1029/2004WR003347).

15. Qi, R., et al. Design of carbon dioxide storage in a North Sea aquifer using streamline-based simulation. in SPE 109905 proceedings of the SPE Annual Meeting, Anaheim. November 2007. California.

16. Saadatpoor, E., S. Bryant, and K. Sepehrnoori. Effect of Heterogeneous Capillary Pressure on Bouyancy-Driven CO2 Migration. in Improved Oil Recovery Symposium 2008. Tulsa, OKlahoma: SPE 113984, proceedings of the SPE/DOE.

17. Chierici, G.L., G.M. Ciucci, and G. Long, Experimental research on gas saturation behind the water front in gas reservoirs subjected to water drive. Proc. of the World Petroleum Congress, Frankfurt am Main, 1963. sec II, paper 17, PD6: p. 483-498.

18. Crowell, D.C., G.W. Dean, and A.G. Loomis, Efficiency of gas displacement from a water-drive reservoir. 1966, Report of Investigations 6735, Bureau of Mines, San Francisco, CA (USA). San Francisco Petroleum Research Lab.

19. Delclaud, J., Laboratory measurements of the residual gas saturation, in Second European Core Analysis Symposium. May 1991: London.

20. Geffen, T.M., et al., Efficiency of gas displacment from porous media by liquid flooding. AIME 1952. 195: p. $29-38$.

21. Jerauld, G.R., General three-phase relative permeability model for Prudhoe Bay. SPE Reservoir Engineering, 1997. 12(4).

22. Kantzas, A., M. Ding, and J. Lee, Residual gas saturation revisited. SPE. Reservoir Evaluation \& Engineering, 2001. 4(6): p. 467-476.

23. Kleppe, J., et al. Representation of capillary pressure hysteresis in reservoir simulation. in SPE 38899, proceedings of the SPE Annual Meeting. October 1997. San Antonio, Texas.

24. Kralik, J.G., et al. Effect of Trapped Gas on Relative Permeability and Residual Oil Saturation in an Oil-Wet Sandstone. in Annual Technical Conference and Exhibition. 2000. Dallas, Texas, USA., 1-4: SPE 62997, proceedings of the SPE

25. Land, C.S., Calculation of imbibition relative permeability for two-and three-phase flow from rock properties. SPE Journal, 1968. 8(2): p. 149-156.

26. Land, C.S., Comparison of Calculating with Experimental Imbibition Relative Permeability SPE Journal, 1971. 11(4): p. $419-425$.

27. Ma, T.D. and G.K. Youngren. Performance of immiscible water-alternating-gas (IWAG) injection at Kuparuk River Unit, North Slope, Alaska. in SPE 2860, proceedings of the SPE Annual Meeting. September 1994. New Orleans, Louisiana.

28. McKay, B.A., Laboratory studies of gas displacement from sandstone reservoirs having strong water drive. APEA Journal, 1974: p. 189-194.

29. Plug, W.J., Measurements of capillary pressure and electric permittivity of gas-water systems in porous media at elevated pressures. 2007, Delft University.

30. Suzanne, K., G. Hamon, and J. Billiotte. Experiemntal relationships between residual gas saturation and initial gas saturation in heterogeneous sandstone reservoir. in Annual Technical Conference and Exhibition. 2003. Denver, Colorado, USA., 5-8: SPE 84038, proceedings of the SPE.

31. Ma, T.D. and G.K. Youngren. Performance of immiscible water-alternating-gas (IWAG) injection at Kuparuk River Unit, North Slope, Alaska. in SPE Annual Meeting. 1994. New Orleans, Louisiana: SPE 2860, proceedings of the SPE.

32. DiCarlo, D.A., A. Sahni, and M.J. Blunt, Three-Phase Relative permeability of Water-Wet, Oil-Wet and Mixed-Wet Sandpacks. SPE Journal 2000. 5: p. 82-91.

33. Aissaoui, A., Etude théorique et expérimentale de l'hystérésis des pressions capillaries et des perméabilitiés relatives en vue du stockage souterrain de gaz. 1983, Ecole des Mines de Paris: Paris.

34. Valvatne, P.H. and M. Blunt, Predictive pore-scale modeling of two-phase flow in mixed wet media. Water Resources Research, 2004. 40(7): p. W07406 doi: 10.1029/2003WR002627 